\title{
PARTIAL SUMS OF THE GAUSSIAN $q$-BINOMIAL COEFFICIENTS, THEIR RECIPROCALS, SQUARE AND SQUARED RECIPROCALS WITH APPLICATIONS
}

\author{
EMRAH KILIÇ AND ILKER AKKUS \\ Received 22 November, 2017
}

\begin{abstract}
In this paper, we shall derive formulæ for partial sums of the Gaussian $q$-binomial coefficients, their reciprocals, squares and squared reciprocals. To prove the claimed results, we use $q$-calculus. As applications of our results, we give some interesting generalized Fibonomial sums formulæ.
\end{abstract}

2010 Mathematics Subject Classification: 11B65; 05A10; 11B37

Keywords: Fibonomial coefficients, Gaussian $q$-binomial coefficients, sum identities

\section{INTRODUCTION}

For $n>1$, define the second order linear sequences $\left\{U_{n}\right\}$ and $\left\{V_{n}\right\}$ by

$$
\begin{array}{ll}
U_{n}=p U_{n-1}+U_{n-2}, & U_{0}=0, U_{1}=1, \\
V_{n}=p V_{n-1}+V_{n-2}, & V_{0}=2, V_{1}=p .
\end{array}
$$

When $p=1, U_{n}=F_{n}$ ( $n$th Fibonacci number) and $V_{n}=L_{n}$ ( $n$th Lucas number), resp. Falcon and Plaza named the previous sequences as $k$-Fibonacci and $k$-Lucas numbers, see $[5,6]$.

For $n \geq k \geq 1$, define the generalized Fibonomial coefficients by

$$
\left\{\begin{array}{l}
n \\
k
\end{array}\right\}_{U}:=\frac{U_{1} U_{2} \ldots U_{n}}{\left(U_{1} U_{2} \ldots U_{k}\right)\left(U_{1} U_{2} \ldots U_{n-k}\right)}
$$

with $\left\{\begin{array}{l}n \\ 0\end{array}\right\}_{U}=\left\{\begin{array}{l}n \\ n\end{array}\right\}_{U}=1$. When $p=1$, we obtain the usual Fibonomial coefficients, denoted by $\left\{\begin{array}{l}n \\ k\end{array}\right\}_{F}$. For more details about the Fibonomial and generalized Fibonomial coefficients, see $[7,9,22]$.

The Binet forms are

$$
U_{n}=\frac{\alpha^{n}-\beta^{n}}{\alpha-\beta} \text { and } V_{n}=\alpha^{n}+\beta^{n},
$$

where $\alpha, \beta=\left(p \pm \sqrt{p^{2}+4}\right) / 2$. 
Throughout this paper we will use the following notations: the $q$-Pochhammer symbol $(x ; q)_{n}=(1-x)(1-x q) \cdots\left(1-x q^{n-1}\right)$ and the Gaussian $q$-binomial coefficients

$$
\left[\begin{array}{l}
n \\
k
\end{array}\right]_{q}=\frac{(q ; q)_{n}}{(q ; q)_{k}(q ; q)_{n-k}} .
$$

The link between the generalized Fibonomial and Gaussian $q$-binomial coefficients is

$$
\left\{\begin{array}{l}
n \\
k
\end{array}\right\}_{U}=\alpha^{k(n-k)}\left[\begin{array}{l}
n \\
k
\end{array}\right]_{q} \text { with } q=-\alpha^{-2} .
$$

By taking $q=\beta / \alpha$, the Binet formulæ are reduced to the following forms:

$$
U_{n}=\alpha^{n-1} \frac{1-q^{n}}{1-q} \text { and } V_{n}=\alpha^{n}\left(1+q^{n}\right),
$$

where $\mathbf{i}=\sqrt{-1}=\alpha \sqrt{q}$. For later use note that $q$-form of the coefficient $p$ in the recurrence relations of $\left\{U_{n}\right\}$ and $\left\{V_{n}\right\}$ is $(1+q)(-q)^{-1 / 2}$.

The Fibonomial coefficients surprisingly appear in several places in the literature (for more details, we refer to $[4,10,11,17,18]$ ). Nowadays interesting sums including the Fibonomial coefficients with certain factors or sign functions have been introduced and computed by several authors (see [12-16, 19-21,23]).

Marques and Trojovsky [19] presented some Fibonomial sums formulæ with the Fibonacci and Lucas numbers as coefficients. For example, for positive integers $m$ and $n$, they showed that

$$
\sum_{j=0}^{4 m+2}(-1)^{\frac{j(j-1)}{2}}\left\{\begin{array}{c}
4 m \\
j
\end{array}\right\}_{F} F_{n+4 m-j}=\frac{1}{2} F_{2 m+n} \sum_{j=0}^{4 m}(-1)^{\frac{j(j-1)}{2}}\left\{\begin{array}{c}
4 m \\
j
\end{array}\right\}_{F} L_{2 m-j} .
$$

Kıliç and Prodinger [14] gave a systematic approach to compute certain sums of squares of Fibonomial coefficients with finite products of generalized Fibonacci and Lucas numbers as coefficients. For example, if $n$ is nonnegative integer, then they proved the following Gaussian $q$-binomial sums identity

$$
\begin{aligned}
& \sum_{k=0}^{2 n+1}\left[\begin{array}{c}
2 n+1 \\
k
\end{array}\right]_{q}^{2}(-1)^{k} q^{k^{2}-2 k n-3 k}\left(1-q^{2 k}\right)^{2} \\
& \quad=2(-1)^{n+1} q^{-n^{2}-2 n-2} \frac{(1+q)\left(1-q^{2 n+1}\right)\left(1-q^{2 n+1}\right)}{\left(1+q^{2 n}\right)}\left[\begin{array}{c}
2 n+1 \\
n
\end{array}\right]_{q^{2}} .
\end{aligned}
$$


Recently Marques and Trojovsky [20] derived various interesting Fibonomial sums formulæ with certain weight functions. For example, they gave that for any nonnegative integers $l$ and $n$,

$$
\sum_{j=0}^{4 l+3} \operatorname{sgn}(2 l+1-j)\left\{\begin{array}{c}
4 l+3 \\
j
\end{array}\right\}_{F} F_{n-j}=\frac{F_{2 l}}{F_{4 l+3}}\left\{\begin{array}{c}
4 l+3 \\
2 l+1
\end{array}\right\}_{F} F_{n-4 l-3}
$$

and

$$
\sum_{j=0}^{4 l+1} \operatorname{sgn}(2 l-j)\left\{\begin{array}{c}
4 l+1 \\
j
\end{array}\right\}_{F} F_{n-j}=-\frac{F_{2 l-1}}{F_{4 l+1}}\left\{\begin{array}{c}
4 l+1 \\
2 l
\end{array}\right\}_{F} F_{n-4 l-1},
$$

where $\operatorname{sgn}(x)$ denotes the sign function of $x$, defined by

$$
\operatorname{sgn}(x)=\left\{\begin{array}{cc}
\frac{x}{|x|} & \text { if } x \neq 0 \\
0 & \text { if } x=0
\end{array}\right.
$$

Much recently Kılıç and Akkuş [1] generalized all the results of [20] through the Gaussian $q$-binomial coefficients instead of the Fibonomial coefficients with additional parameters. They also gave analogues of all the sums formulæ whose upper bounds are even integers. The authors proved the claimed results by mainly and analytically $q$-calculus, and the celebrated Zeilberger algorithm for some steps of their proofs. For convenience of the readers, we recall two sums formulæ from [1]:

$$
\begin{aligned}
& \sum_{j=0}^{4 l+3} \operatorname{sgn}(2 l+1-j)\left[\begin{array}{c}
4 l+3 \\
j
\end{array}\right]_{q}(-1)^{\frac{1}{2} j(j-2)} q^{-\frac{1}{2} j(4 l-j+2)}\left(1-q^{n-j}\right) z^{[2 \nmid j]} \\
& =q^{2-2 l^{2}} \frac{1-q^{2 l}}{1-q^{4 l+3}}\left[\begin{array}{c}
4 l+3 \\
2 l+1
\end{array}\right]_{q}\left(1-q^{n-4 l-3}\right),
\end{aligned}
$$

and

$$
\begin{aligned}
& \sum_{j=0}^{4 l+1} \operatorname{sgn}(2 l-j)\left[\begin{array}{c}
4 l+1 \\
j
\end{array}\right]_{q}(-1)^{\frac{1}{2} j^{2}} q^{\frac{1}{2} j(j-4 l)}\left(1-q^{n-j}\right) z^{[2 \mid j]} \\
& =-(-q)^{\frac{4 l+3-4 l^{2}}{2}} \frac{1-q^{2 l-1}}{1-q^{4 l+1}}\left[\begin{array}{c}
4 l+1 \\
2 l
\end{array}\right]_{q}\left(1-q^{n-4 l-1}\right),
\end{aligned}
$$

where $z=(1+q)(-q)^{-1 / 2}$, resp. If one take $q=\left(p-\sqrt{p^{2}+4}\right) /\left(p+\sqrt{p^{2}+4}\right)$, then these sums are reduced to the following generalized Fibonomial sums formulæ: For nonnegative integers $l$ and $n$

$$
\sum_{j=0}^{4 l+3} \operatorname{sgn}(2 l+1-j) p^{[2 \nmid j]}\left\{\begin{array}{c}
4 l+3 \\
j
\end{array}\right\}_{U} U_{n-j}=\frac{U_{2 l}}{U_{4 l+3}}\left\{\begin{array}{c}
4 l+3 \\
2 l+1
\end{array}\right\}_{U} U_{n-4 l-3},
$$


and

$$
\sum_{j=0}^{4 l+1} \operatorname{sgn}(2 l-j) p^{[2 \mid j]}\left\{\begin{array}{c}
4 l+1 \\
j
\end{array}\right\}_{U} U_{n-j}=-\frac{U_{2 l-1}}{U_{4 l+1}}\left\{\begin{array}{c}
4 l+1 \\
2 l
\end{array}\right\}_{U} U_{n-4 l-1},
$$

where [ ] stands for the Iverson notation (see [8]). We would like to take attention of the readers to factors $p^{[2 \mid j]}$ and $p^{[2 \nmid j]}$ in these generalizations just above. These are not easily seen while deriving the generalized sums formulæ. Indeed, when $p=$ 1, these generalized Fibonomial sums formulæ are reduced to the sums formulæ (1.1) and (1.2) given in [20]. Similarly when $q=(1+\sqrt{5}) /(1-\sqrt{5})$ or equivalently $p=1$, some of the results of [1] cover the results of [20].

Othsuka conjectured the following two advanced problems, H-764 and H-768 (see $[2,3])$. Here we recall these problems:

Advanced Problem H-764: For $n \geq 1$, prove that

$$
\begin{aligned}
& \text { (i) } \sum_{k=0}^{n} F_{2(n-k)}\left\{\begin{array}{c}
2 n \\
k
\end{array}\right\}_{F}=\frac{F_{n} F_{n+1}}{F_{2 n-1}}\left\{\begin{array}{c}
2 n \\
n
\end{array}\right\}_{F}, \\
& \text { (ii) } \sum_{k=0}^{n} F_{2(n-k)}\left\{\begin{array}{c}
2 n \\
k
\end{array}\right\}_{F}^{2}=\frac{F_{n}}{L_{n}}\left\{\begin{array}{c}
2 n \\
n
\end{array}\right\}_{F}^{2} .
\end{aligned}
$$

Advanced Problem H-768: For $n \geq 1$, prove that

$$
\begin{aligned}
& \text { (i) } \sum_{k=0}^{n} F_{2(n-k)}\left\{\begin{array}{c}
2 n \\
k
\end{array}\right\}_{F}^{-1}=\frac{F_{2 n+1}\left(F_{2 n+2}+1\right)}{F_{2 n+3}}-\frac{F_{n+1} F_{n+3}}{F_{2 n+3}}\left\{\begin{array}{c}
2 n \\
n
\end{array}\right\}_{F}^{-1}, \\
& \text { (ii) } \sum_{k=0}^{n} F_{2(n-k)}\left\{\begin{array}{c}
2 n \\
k
\end{array}\right\}_{F}^{-2}=\frac{F_{2 n+1}^{2}}{F_{2 n+2}}-\frac{F_{n+1}}{L_{n+1}}\left\{\begin{array}{c}
2 n \\
n
\end{array}\right\}_{F}^{2} .
\end{aligned}
$$

In this paper, inspired by the results of [1] and earlier partial $q$-binomial sums formulæ, we shall derive new kinds interesting partial sums formulæ including the Gaussian $q$-binomial coefficients which are completely different from the sums formulæ given in [1]. We summarize what we present in this paper below.

- Sums of half of the Gaussian $q$-binomial coefficients.

- Partial sums of square of the Gaussian $q$-binomial coefficients.

- Partial sums of reciprocals of the Gaussian $q$-binomial coefficients.

- Partial sums of squared reciprocals of the Gaussian $q$-binomial coefficients.

All above sums will be computed with certain weight functions. Further we notice that special cases of our results give us solutions for Advanced Problems H-764 and $\mathrm{H}-768$ in $[2,3]$. All the identities and formulæ we will obtain hold for general $q$, and results about Fibonomial and Fibonacci numbers come out as corollaries for the 
special choice of $q$. One could derive many special corollaries by choosing special $q$ values.

\section{UPPER BOUND CASES}

Now we present our results. Before this, we give an auxiliary lemma and then give one of our main results.

Lemma 1. For nonnegative integer $n$, any nonzero constant $c$ and any function $f$,

(i)

$$
\sum_{j=0}^{n} c^{[2 \nmid j]} f(j)=\sum_{j=0}^{n}\left(\frac{c+1-(c-1)(-1)^{j}}{2}\right) f(j),
$$

(ii)

$$
\sum_{j=0}^{n} c^{[2 \mid j]} f(j)=\sum_{j=0}^{n}\left(\frac{c+1+(c-1)(-1)^{j}}{2}\right) f(j),
$$

where [ ] stands for the Iverson notation.

Proof. For any integer $j$, since $[2 \mid j]=\left(1+(-1)^{j}\right) / 2$ and

$$
\begin{aligned}
c^{[2 \nmid j]} f(j) & =[2 \mid j] f(j)+c[2 \nmid j] f(j)=([2 \mid j]+c(1-[2 \mid j])) f(j) \\
& =(c-(c-1)[2 \mid j]) f(j)=\left(c+1-(c-1)(-1)^{j}\right) f(j) / 2,
\end{aligned}
$$

the first claim (i) follows. The latter is similarly proven.

Theorem 1. (i) For even $n$,

$$
\begin{aligned}
& \sum_{j=0}^{n}\left[\begin{array}{c}
2 n \\
j
\end{array}\right]_{q} \mathbf{i}^{j^{2}}(-1)^{j(n-1)} q^{\frac{j(j-2 n+2)}{2}}\left(1-q^{2 n-2 j}\right) z^{[2 \nmid j]} \\
&=(-1)^{n+1} \mathbf{i}^{-n^{2}} q^{-\frac{1}{2} n^{2}+n-1} \frac{\left(1-q^{n}\right)\left(1-q^{n+1}\right)}{1-q^{2 n-1}}\left[\begin{array}{c}
2 n \\
n
\end{array}\right]_{q},
\end{aligned}
$$

(ii) For odd n,

$$
\begin{aligned}
\sum_{j=0}^{n}\left[\begin{array}{c}
2 n \\
j
\end{array}\right]_{q} \mathbf{i}^{j^{2}}(-1)^{j(n-1)} & \left(1-q^{2 n-2 j}\right) z^{[2 \mid j]} \\
& =(-1)^{n+1} \mathbf{i}^{-n^{2}} q^{-\frac{1}{2} n^{2}+n-1} \frac{\left(1-q^{n}\right)\left(1-q^{n+1}\right)}{1-q^{2 n-1}}\left[\begin{array}{c}
2 n \\
n
\end{array}\right]_{q}
\end{aligned}
$$

where $z=-\mathbf{i} q^{-1 / 2}(1+q)$. 
Proof. We prove the claim (i). The latter is similar. Since $n$ is even, say $n=2 k$, then we have to prove that

$$
\begin{gathered}
\sum_{j=0}^{2 k}\left[\begin{array}{c}
4 k \\
j
\end{array}\right]_{q}(-q)^{\frac{j(j-4 k+2)}{2}}\left(1-q^{4 k-2 j}\right)\left(\frac{z+1-(z-1)(-1)^{j}}{2}\right) \\
=(-q)^{-2 k^{2}+2 k-1} \frac{\left(1-q^{2 k}\right)\left(1-q^{2 k+1}\right)}{\left(1-q^{4 k-1}\right)}\left[\begin{array}{c}
4 k \\
2 k
\end{array}\right]_{q}
\end{gathered}
$$

Note that for any functions $F(j)$ and $G(j)$ of $j$, the following equality holds

$$
\sum_{j=0}^{2 n}[F(j)-G(j)]=\sum_{j=0}^{n}[F(2 j)-G(2 j)]+\sum_{j=0}^{n-1}[F(2 j+1)-G(2 j+1)] .
$$

By this fact, we rewrite the LHS of the claim mentioned above as

$$
\begin{aligned}
\sum_{j=0}^{2 k}\left[\begin{array}{c}
4 k \\
j
\end{array}\right]_{q}(-q)^{\frac{j(j-4 k+2)}{2}}\left(1-q^{4 k-2 j}\right)\left(\frac{z+1-(z-1)(-1)^{j}}{2}\right) \\
=\frac{1}{2} \sum_{j=0}^{2 k}\left[\begin{array}{c}
4 k \\
j
\end{array}\right]_{q}(-q)^{\frac{j(j-4 k+2)}{2}}\left(1-q^{4 k-2 j}\right)\left(z+1-(z-1)(-1)^{j}\right) \\
=\frac{(z+1)}{2} \sum_{j=0}^{k}\left[\begin{array}{c}
4 k \\
2 j
\end{array}\right]_{q} q^{2 j(j-2 k+1)}\left(1-q^{4 k-4 j}\right) \\
\quad-\frac{(z-1)}{2} \sum_{j=0}^{k}\left[\begin{array}{c}
4 k \\
2 j
\end{array}\right]_{q} q^{2 j(j-2 k+1)}\left(1-q^{4 k-4 j}\right) \\
\quad+\frac{1}{2} \sum_{j=0}^{k-1}\left[\begin{array}{c}
4 k \\
2 j+1
\end{array}\right]_{q}(-q)^{\frac{(2 j+1)(2 j-4 k+3)}{2}}\left(1-q^{4 k-4 j-2}\right)(z+1) \\
\quad+\frac{1}{2} \sum_{j=0}^{k-1}\left[\begin{array}{c}
4 k \\
2 j+1
\end{array}\right]_{q}(-q)^{\frac{(2 j+1)(2 j-4 k+3)}{2}}\left(1-q^{4 k-4 j-2}\right)(z-1) \\
=\sum_{j=0}^{k}\left[\begin{array}{c}
4 k \\
2 j
\end{array}\right]_{q}^{2 j(j-2 k+1)\left(1-q^{4 k-4 j}\right)} \\
\quad+z \sum_{j=0}^{k-1}\left[\begin{array}{c}
4 k \\
2 j+1
\end{array}\right]_{q}(-q)^{\frac{(2 j+1)(2 j-4 k+3)}{2}}\left(1-q^{4 k-4 j-2}\right),
\end{aligned}
$$


which, by the identity

$$
\left[\begin{array}{l}
n+1 \\
k+1
\end{array}\right]_{q}=\frac{1-q^{n-k+1}}{1-q^{k+1}}\left[\begin{array}{c}
n+1 \\
k
\end{array}\right]_{q},
$$

equals

$$
\begin{aligned}
\sum_{j=0}^{k} & {\left[\begin{array}{l}
4 k \\
2 j
\end{array}\right]_{q} q^{2 j(j-2 k+1)}\left(1-q^{4 k-4 j}\right) } \\
& +\sum_{j=0}^{k-1}\left[\begin{array}{l}
4 k \\
2 j
\end{array}\right]_{q}(-q)^{\frac{(2 j+1)(2 j-4 k+3)}{2}}\left(1-q^{4 k-4 j-2}\right) \frac{\left(1-q^{4 k-2 j}\right)}{1-q^{2 j+1}} z \\
= & \sum_{j=0}^{k-1}\left[\begin{array}{l}
4 k \\
2 j
\end{array}\right]_{q} q^{2 j(j-2 k+1)}\left(1-q^{4 k-4 j}\right) \\
& +\sum_{j=0}^{k-1}\left[\begin{array}{l}
4 k \\
2 j
\end{array}\right]_{q}(-q)^{\frac{(2 j+1)(2 j-4 k+3)}{2}}\left(1-q^{4 k-4 j-2}\right) \frac{\left(1-q^{4 k-2 j}\right)}{1-q^{2 j+1}} z \\
= & \frac{-q^{1+2 k-2 k^{2}}(q ; q)_{4 k}}{\left(-q+q^{4 k}\right)(q ; q)_{2 k}(q ; q)_{2 k-2}}+\frac{q^{2 k-2 k^{2}}(1+q)(q ; q)_{4 k}}{\left(-q+q^{4 k}\right)(q ; q)_{2 k-1}^{2}}
\end{aligned}
$$

which, by the definition of the Gaussian $q$-binomial coefficients, equals

$$
\begin{aligned}
& q^{2 k-2 k^{2}} \frac{\left(1-q^{2 k-1}\right)\left(1-q^{2 k}\right)}{1-q^{4 k-1}}\left[\begin{array}{l}
4 k \\
2 k
\end{array}\right]_{q} \\
& -q^{2 k-2 k^{2}-1}(1+q) \frac{\left(1-q^{2 k}\right)\left(1-q^{2 k+1}\right)}{1-q^{4 k-1}}\left[\begin{array}{c}
4 k \\
2 k-1
\end{array}\right]_{q} \\
& =q^{2 k-2 k^{2}} \frac{\left(1-q^{2 k-1}\right)\left(1-q^{2 k}\right)}{1-q^{4 k-1}}\left[\begin{array}{c}
4 k \\
2 k
\end{array}\right]_{q}-q^{2 k-2 k^{2}-1}(1+q) \frac{\left(1-q^{2 k}\right)^{2}}{1-q^{4 k-1}}\left[\begin{array}{c}
4 k \\
2 k
\end{array}\right]_{q} \\
& =q^{2 k-2 k^{2}-1} \frac{1-q^{2 k}}{1-q^{4 k-1}}\left[\begin{array}{c}
4 k \\
2 k
\end{array}\right]_{q}\left[q\left(1-q^{2 k-1}\right)-(1+q)\left(1-q^{2 k}\right)\right] \\
& =-q^{2 k-2 k^{2}-1} \frac{\left(1-q^{2 k}\right)\left(1-q^{2 k+1}\right)}{1-q^{4 k-1}}\left[\begin{array}{c}
4 k \\
2 k
\end{array}\right]_{q},
\end{aligned}
$$

as claimed for even $n$ such that $n=2 k$. The other claim is similarly proven.

By taking $q=\left(p-\sqrt{p^{2}+4}\right) /\left(p+\sqrt{p^{2}+4}\right)$ in Theorem 1 , we have the following result. 
Corollary 1. (i)

For odd $n$,

(ii)

$$
\sum_{j=0}^{n}\left\{\begin{array}{c}
2 n \\
j
\end{array}\right\}_{U} p^{[2 \mid j]} U_{2 n-2 j}=\frac{U_{n} U_{n+1}}{U_{2 n-1}}\left\{\begin{array}{c}
2 n \\
n
\end{array}\right\}_{U} .
$$

For even $n$,

$$
\sum_{j=0}^{n}\left\{\begin{array}{c}
2 n \\
j
\end{array}\right\}_{U} p^{[2 \nmid j]} U_{2 n-2 j}=\frac{U_{n} U_{n+1}}{U_{2 n-1}}\left\{\begin{array}{c}
2 n \\
n
\end{array}\right\}_{U} .
$$

We notice that when $p=1$, the results of Corollary 1 cover solutions for the advanced problem 764(i) [2].

Theorem 2. For nonnegative integers $n$ and $m$,

$$
\sum_{j=0}^{m}\left[\begin{array}{l}
n \\
j
\end{array}\right]_{q}^{2} q^{j(j-n+1)}\left(1-q^{n-2 j}\right)=q^{m(m-n+1)}\left(1-q^{n-m}\right)\left[\begin{array}{l}
n \\
m
\end{array}\right]_{q}\left[\begin{array}{c}
n-1 \\
m
\end{array}\right]_{q} .
$$

Proof. Denote the LHS of the claim by $F(n, m)$, that is,

$$
F(n, m)=\sum_{j=0}^{m}\left[\begin{array}{l}
n \\
j
\end{array}\right]_{q}^{2} q^{j(j-n+1)}\left(1-q^{n-2 j}\right) .
$$

For $m \geq n, F(n, m)$ is a whole sum and equals 0 . To see this fact, consider

$$
F(n, m)=\sum_{j \geq 0}\left[\begin{array}{l}
n \\
j
\end{array}\right]_{q}^{2} q^{j(j-n+1)}\left(1-q^{n-2 j}\right),
$$

which, by taking $n-j$ instead of $j$, gives us

$$
F(n, m)=\sum_{j \geq 0}\left[\begin{array}{l}
n \\
j
\end{array}\right]_{q}^{2} q^{(j-n)(j-1)}\left(1-q^{2 j-n}\right)=-F(n, m),
$$

which gives us $F(n, m)=0$, as claimed.

Define

$$
G(n, m)=q^{m(m-n+1)}\left(1-q^{n-m}\right)\left[\begin{array}{l}
n \\
m
\end{array}\right]_{q}\left[\begin{array}{c}
n-1 \\
m
\end{array}\right]_{q} .
$$

Then we have

$$
G(n, m)=F(n, m),
$$

which follows from 


$$
\begin{aligned}
& G(n, j)-G(n, j-1) \\
& =q^{j(j-n+1)}\left(1-q^{n-j}\right)\left[\begin{array}{l}
n \\
j
\end{array}\right]_{q}\left[\begin{array}{c}
n-1 \\
j
\end{array}\right]_{q}-q^{(j-1)(j-n)}\left(1-q^{n-j+1}\right)\left[\begin{array}{c}
n \\
j-1
\end{array}\right]_{q}\left[\begin{array}{c}
n-1 \\
j-1
\end{array}\right]_{q} \\
& =q^{j(j-n+1)}\left[\begin{array}{l}
n \\
j
\end{array}\right]_{q}\left(\left(1-q^{n-j}\right) \frac{(q ; q)_{n-1}}{(q ; q)_{j}(q ; q)_{n-j-1}}-\frac{q^{n-2 j}\left(1-q^{j}\right)^{2}(q ; q)_{n-1}}{\left(1-q^{n-j}\right)(q ; q)_{j}(q ; q)_{n-j-1}}\right) \\
& =q^{j(j-n+1)}\left[\begin{array}{l}
n \\
j
\end{array}\right]_{q}\left(\frac{\left(1-q^{n-j}\right)^{2}}{1-q^{c n}}\left[\begin{array}{l}
n \\
j
\end{array}\right]_{q}-\frac{q^{n-2 j}\left(1-q^{j}\right)^{2}}{1-q^{n}}\left[\begin{array}{l}
n \\
j
\end{array}\right]_{q}\right) \\
& =q^{j(j-n+1)}\left[\begin{array}{l}
n \\
j
\end{array}\right]_{q}^{2}\left(\frac{\left(1-q^{n-j}\right)^{2}-q^{n-2 j}\left(1-q^{j}\right)^{2}}{1-q^{n}}\right) \\
& =q^{j(j-n+1)}\left[\begin{array}{l}
n \\
j
\end{array}\right]_{q}^{2}\left(\frac{\left(1-q^{n-2 j}\right)\left(1-q^{n}\right)}{1-q^{n}}\right) \\
& =q^{j(j-n+1)}\left(1-q^{n-2 j}\right)\left[\begin{array}{l}
n \\
j
\end{array}\right]_{q}^{2}
\end{aligned}
$$

as claimed.

As special cases of Theorem 2 with $q=\left(p-\sqrt{p^{2}+4}\right) /\left(p+\sqrt{p^{2}+4}\right), " m \rightarrow$ $n, n \rightarrow 2 n$ " and " $m \rightarrow n, n \rightarrow 2 n$ ", we have the following result, resp.

Corollary 2. For nonnegative integer $n$,

$$
\sum_{j=0}^{n}\left\{\begin{array}{c}
2 n \\
j
\end{array}\right\}_{U}^{2} U_{2 n-2 j}=\frac{U_{n}}{V_{n}}\left\{\begin{array}{c}
2 n \\
n
\end{array}\right\}_{U}^{2} \text { and } \sum_{j=0}^{2 n} U_{2 n-2 j}\left\{\begin{array}{c}
2 n \\
j
\end{array}\right\}_{U}^{2}=0 .
$$

When $p=1$, the first result of Corollary 2 gives us a solution for the advanced problem 764(ii) [2].

Now we present a sum formula for the squared reciprocals of the Gaussian $q$ binomial coefficient without proof.

Theorem 3. For nonnegative integers $n$ and $m$,

$$
\sum_{k=0}^{m}\left[\begin{array}{l}
n \\
k
\end{array}\right]_{q}^{-2} q^{k(n+1-k)}\left(1-q^{n-2 k}\right)=\frac{\left(1-q^{n+1}\right)^{2}}{1-q^{n+2}}-q^{(m+1)(n-m)} \frac{\left(1-q^{m+1}\right)^{2}}{\left(1-q^{n+2}\right)}\left[\begin{array}{l}
n \\
m
\end{array}\right]_{q}^{-2} .
$$

As special cases of Theorem 3 with $q=\left(p-\sqrt{p^{2}+4}\right) /\left(p+\sqrt{p^{2}+4}\right), " m \rightarrow$ $n, n \rightarrow 2 n$ " and " $m \rightarrow n, n \rightarrow 2 n$ ", we have the following result, resp. 
Corollary 3. For nonnegative integer $n$,

$$
\sum_{j=0}^{n} U_{2 n-2 j}\left\{\begin{array}{c}
2 n \\
j
\end{array}\right\}_{U}^{-2}=\frac{U_{2 n+1}^{2}}{U_{2 n+2}}-\frac{U_{n+1}}{V_{n+1}}\left\{\begin{array}{c}
2 n \\
n
\end{array}\right\}_{U}^{-2} \text { and } \sum_{j=0}^{2 n} U_{2 n-2 j}\left\{\begin{array}{c}
2 n \\
j
\end{array}\right\}_{U}^{-2}=0 .
$$

When $p=1$, the first result of Corollary 3 gives a solution for the advanced problem 768(ii) [3].

\section{AdDitionAl SUMS FORMULÆ}

In this Section, we will give new partial sums formulæ including the Gaussian $q$-binomial coefficients and their reciprocals.

Theorem 4. For nonnegative integers $n$ and $m$,

(i)

$$
\sum_{j=0}^{m}\left[\begin{array}{l}
n \\
j
\end{array}\right]_{q} q^{j(j+1-n)}\left(1-q^{n-2 j}\right)=q^{m(m-n+1)}\left(1-q^{n-m}\right)\left[\begin{array}{l}
n \\
m
\end{array}\right]_{q}
$$

(ii)

$\sum_{j=0}^{m}\left[\begin{array}{l}n \\ j\end{array}\right]_{q}^{-1} q^{j(n+1-j)}\left(1-q^{n-2 j}\right)=\left(1-q^{n+1}\right)-q^{(m+1)(n-m)}\left(1-q^{m+1}\right)\left[\begin{array}{l}n \\ m\end{array}\right]_{q}^{-1}$.

Proof. As a showcase, we only prove the second formula. Denote

$$
F(n, m)=\sum_{j=0}^{m}\left[\begin{array}{l}
n \\
j
\end{array}\right]_{q}^{-1} q^{j(n+1-j)}\left(1-q^{n-2 j}\right) .
$$

For $m \geq n, F(n, m)$ is a whole sum and equals 0 . To see this, consider by taking $n-j$ instead of $j$,

$$
\begin{aligned}
F(n, m) & =\sum_{j \geq 0}\left[\begin{array}{l}
n \\
j
\end{array}\right]_{q}^{-1} q^{j(n+1-j)}\left(1-q^{n-2 j}\right) \\
& =\sum_{j \geq 0}\left[\begin{array}{l}
n \\
j
\end{array}\right]_{q}^{-1} q^{(n-j)(j+1)}\left(1-q^{2 j-n}\right) \\
& =-F(n, m) .
\end{aligned}
$$

So we get $F(n, m)=0$.

Define

$$
G(n, m)=\left(1-q^{n+1}\right)-q^{(m+1)(n-m)}\left(1-q^{m+1}\right)\left[\begin{array}{c}
n \\
m
\end{array}\right]_{q}^{-1}
$$


Then we have

which follows from

$$
G(n, m)=F(n, m),
$$

$$
\begin{aligned}
& G(n, j)-G(n, j-1) \\
& =\left(1-q^{n+1}\right)-q^{(j+1)(n-j)}\left(1-q^{j+1}\right)\left[\begin{array}{l}
n \\
j
\end{array}\right]_{q}^{-1} \\
& -\left(\left(1-q^{n+1}\right)-q^{j(n-j+1)}\left(1-q^{j}\right)\left[\begin{array}{c}
n \\
j-1
\end{array}\right]_{q}^{-1}\right) \\
& =-q^{(j+1)(n-j)}\left(1-q^{j+1}\right)\left[\begin{array}{l}
n \\
j
\end{array}\right]_{q}^{-1}+q^{j(n-j+1)}\left(1-q^{j}\right)\left[\begin{array}{c}
n \\
j-1
\end{array}\right]_{q}^{-1} \\
& =-q^{(j+1)(n-j)}\left(1-q^{j+1}\right)\left[\begin{array}{l}
n \\
j
\end{array}\right]_{q}^{-1}+q^{j(n-j+1)}\left(1-q^{j}\right) \frac{\left(1-q^{n+1-j}\right)}{\left(1-q^{j}\right)}\left[\begin{array}{l}
n \\
j
\end{array}\right]_{q}^{-1} \\
& =q^{j-j^{2}+j n}\left[q^{-2 j+n}\left(-1+q^{j+1}\right)+\left(1-q^{n+1-j}\right)\right]\left[\begin{array}{l}
n \\
j
\end{array}\right]_{q}^{-1} \\
& =q^{j(n+1-j)}\left(1-q^{n-2 j}\right)\left[\begin{array}{l}
n \\
j
\end{array}\right]_{q}^{-1},
\end{aligned}
$$

as claimed.

\section{REFERENCES}

[1] E. Kılıç and I. Akkus, "On Fibonomial sums identities with special sign functions: analytically q-calculus approach.” Mathematica Slovaca, vol. 68, no. 3, pp. 501-518, 2018.

[2] H. Ohtsuka, "Advanced Problem H-764." Fibonacci Q., vol. 52, no. 4, p. 375, 2014.

[3] H. Ohtsuka, "Advanced Problem H-768." Fibonacci Q., vol. 53, no. 1, p. 89, 2015.

[4] L. Carlitz, "The characteristic polynomial of a certain matrix of binomial coefficients." Fibonacci $Q$. , vol. 3, pp. 81-89, 1965.

[5] S. Falcon, "On the $k$-Lucas numbers." Int. J. Contemp. Math. Sci., vol. 6, no. 21-24, pp. 10391050, 2011.

[6] S. Falcón and A. Plaza, "On the Fibonacci $k$-numbers." Chaos Solitons Fractals, vol. 32, no. 5, pp. 1615-1624, 2007, doi: 10.1016/j.chaos.2006.09.022.

[7] H. Gould, "The bracket function and Fontené-Ward generalized binomial coefficients with application to Fibonomial coefficients." Fibonacci Q., vol. 7, pp. 23-40, 1969.

[8] R. L. Graham, D. E. Knuth, and O. Patashnik, Concrete mathematics: a foundation for computer science. 2nd ed., 2nd ed. Amsterdam: Addison-Wesley Publishing Group, 1994.

[9] V. E. Hoggatt, "Fibonacci numbers and generalized binomial coefficients." Fibonacci Q., vol. 5, pp. 383-400, 1967.

[10] D. Jarden, "Recurring sequences.” Math. Stud., vol. 16, pp. 28-30, 1949. 
[11] E. K1lıç, “The generalized Fibonomial matrix.” Eur. J. Comb., vol. 31, no. 1, pp. 193-209, 2010, doi: 10.1016/j.ejc.2009.03.041.

[12] E. K1lıç, "Evaluation of sums containing triple aerated generalized Fibonomial coefficients." Math. Slovaca, vol. 67, no. 2, pp. 355-370, 2017, doi: 10.1515/ms-2016-0272.

[13] E. Kılıç, I. Akkus, and H. Ohtsuka, "Some generalized Fibonomial sums related with the Gaussian $q$-binomial sums." Bull. Math. Soc. Sci. Math. Roum., Nouv. Sér., vol. 55, no. 1, pp. 51-61, 2012.

[14] E. Kilıç and H. Prodinger, "Closed form evaluation of sums containing squares of Fibonomial coefficients." Math. Slovaca, vol. 66, no. 3, pp. 757-765, 2016, doi: 10.1515/ms-2015-0177.

[15] E. Kılıç and H. Prodinger, "Evaluation of sums involving Gaussian $q$-binomial coefficients with rational weight functions." Int. J. Number Theory, vol. 12, no. 2, pp. 495-504, 2016, doi: 10.1142/S1793042116500305.

[16] E. Kılıç, H. Prodinger, I. Akkus, and H. Ohtsuka, "Formulas for fibonomial sums with generalized Fibonacci and Lucas coefficients." Fibonacci Q., vol. 49, no. 4, pp. 320-329, 2011.

[17] E. Kilıç, G. N. Stănică, and P. Stănică, "Spectral properties of some combinatorial matrices." Congr. Numerantium, vol. 201, pp. 223-235, 2010.

[18] E. Kılıç and P. Stănică, "Generating matrices of $C$-nomial coefficients and their spectra." in Proceedings of the 14th international conference on Fibonacci numbers and their applications, Morelia, Mexico, July 5-9, 2010. México: Sociedad Matemática Mexicana, 2011, pp. 139-154.

[19] D. Marques and P. Trojovský, "On some new sums of Fibonomial coefficients." Fibonacci Q., vol. 50, no. 2, pp. 155-162, 2012.

[20] D. Marques and P. Trojovský, "On some new identities for the Fibonomial coefficients." Math. Slovaca, vol. 64, no. 4, pp. 809-818, 2014, doi: 10.2478/s12175-014-0241-7.

[21] J. Seibert and P. Trojovský, "On some identities for the Fibonomial coefficients." Math. Slovaca, vol. 55, no. 1, pp. 9-19, 2005.

[22] R. Torretto and J. Fuchs, "Generalized binomial coefficients." Fibonacci Q., vol. 2, pp. 296-302, 1964.

[23] P. Trojovský, "On some identities for the Fibonomial coefficients via generating function." Discrete Appl. Math., vol. 155, no. 15, pp. 2017-2024, 2007, doi: 10.1016/j.dam.2007.05.003.

Authors' addresses

Emrah Kılıç

TOBB University of Economics and Technology, Mathematics Department, Sögütözü, 06560 Ankara, Turkey

E-mail address: ekilicdetu.edu.tr

Ilker Akkus

Kırıkkale University, Department of Mathematics, Faculty of Science and Arts, 71450 Kırıkkale, Turkey

E-mail address: iakkus.tregmail.com 